\title{
BUOYANCY EFFECTS ON FLOW TRANSITION IN LOW-DENSITY INERTIAL GAS JETS
}

\author{
Kasyap S. Pasumarthi and Ajay K. Agrawal ${ }^{1}$ \\ School of Aerospace and Mechanical Engineering \\ University of Oklahoma, \\ Norman, OK, USA 73019
}

\begin{abstract}
Effects of buoyancy on transition from laminar to turbulent flow are presented for momentum-dominated helium jet injected into ambient air. The buoyancy was varied in a 2.2-sec drop tower facility without affecting the remaining operating parameters. The jet flow in Earth gravity and microgravity was visualized using the rainbow schlieren deflectometry apparatus. Results show significant changes in the flow structure and transition behavior in the absence of buoyancy.
\end{abstract}

\footnotetext{
${ }^{1}$ Corresponding Author

Lloyd G. \& Joyce Austin Presidential Professor and Associate Professor School of Aerospace and Mechanical Engineering 865 Asp Avenue, Room 208 University of Oklahoma, Norman, OK 73019

Phone: (405) 325-1754, Fax: (405) 325-1088, E-mail: aagrawal@ ou.edu
} 


\section{INTRODUCTION}

Transition from laminar to turbulent flow in gas jets is a subject of considerable interest. A transitional jet consists of a laminar potential core near the injector, separated from a downstream turbulent region at the transition plane. The distance from the injector exit to the transition plane or transition length (z) decreases asymptotically with an increase in the jet Reynolds number defined as $\operatorname{Re}=\mathrm{U}_{\mathrm{j}} \mathrm{d} / v$, where $\mathrm{U}_{\mathrm{j}}$ is the mean jet exit velocity, $\mathrm{d}$ is the injector inside diameter, and $v$ is the kinematic viscosity of the injector fluid. Subbarao and Cantwell (1992) found that the flow transition in a helium jet injected into a coflow of air was also affected by the jet Richardson number, $\mathrm{Ri}=\operatorname{gd}\left(\rho_{\infty}-\rho_{\mathrm{j}}\right) / \rho_{\mathrm{j}} \mathrm{U}_{\mathrm{j}}^{2}$, where $g$ is the gravitational acceleration, $\rho_{\infty}$ and $\rho_{\mathrm{j}}$ are, respectively, the free-stream and jet densities. For a given Re, the transition length decreased asymptotically with increasing jet Richardson number. This effect was attributed to the buoyancy-induced acceleration of the jet fluid, giving rise to faster core velocities and hence, a more unstable flow at higher Ri. They proposed that the sudden breakdown to turbulence was governed by highly structured and repeatable vortices formed in the upstream laminar region.

Periodic oscillations resulting in the breakdown to turbulence have also been observed in low-density gas jets at $\mathrm{Ri}<<1.0$ (Kyle and Sreenivasan, 1993; Richards et al., 1996). In these studies, the buoyancy effects were considered negligible because of the small Ri. However, the buoyancy may be important locally, for example, in the lowmomentum region of the jet shear layer. Buoyant acceleration of the low-density fluid in localized regions of the jet may trigger the instability causing periodic flow oscillations and eventually, the breakdown to turbulence. This explanation is supported by the recent 
experiments of Yildirim and Agrawal (2004), who found that the flow oscillation frequency normalized by the buoyancy time scale correlated with the jet Richardson number in inertial low-density jets for $\mathrm{Ri}<0.1$. Furthermore, the transition length normalized by injector inside diameter correlated with the jet Richardson number for a given Re, an observation similar to that of Subbarao and Cantwell (1992) in buoyant jets. Yildirim and Agrawal (2004) utilized different diameter injector tubes to vary the jet Richardson number for a given Re. Besides the limited range of the jet Richardson number achieved, the similarity parameters are not perfectly matched using this approach. In the present study, the microgravity environment of a drop tower facility is used to vary Ri by several orders of magnitude. The operating parameters such as the jet Reynolds number, the jet to ambient density ratio, and the jet momentum thickness (Kyle and Sreenivasan, 1993) remain unchanged in the drop tower and hence, the buoyancy effects can be isolated. The specific objective of this study is to identify if buoyancy affects transition from laminar to turbulent flow in inertial low-density gas jets.

\section{EXPERIMENTAL SETUP}

Experiments utilizing a compact rig were conducted at NASA John H. Glenn Research Center's 2.2s drop tower facility to simulate microgravity. The flow system comprised of on-board compressed helium gas cylinders, a calibrated mass flow meter, a needle valve to set the flowrate, a solenoid valve to initiate and terminate the flow, and a vertically oriented stainless steel tube to serve as the injector. Yildirim and Agrawal (2004) have provided details of the schlieren apparatus. The color schlieren images are acquired by a high-speed digital camera operated at exposure time of $297-\mu$ s and acquisition rate of 1000 images per second, providing spatial resolution of $0.2 \mathrm{~mm}$. 
The jet flow is initiated and allowed to develop into oscillatory mode in Earth gravity. After storing 0.4-sec of Earth gravity images, the drop rig is released to simulate microgravity during the free fall. The camera stores schlieren images during the 2.2-sec drop. After the drop, the rig is retrieved and the image data from camera memory are transferred to an external hard drive for analysis. Details of the experimental and data analysis procedures are provided by Pasumarthi (2004).

\section{RESULTS AND DISCUSSION}

Although six different experiments were conducted, results are presented only for two test cases at jet Reynolds number of 800 to depict the overall trends. Jet tubes with $\mathrm{d}=14.5 \mathrm{~mm}$ (case 1) and $10.5 \mathrm{~mm}$ (case 2) were used to obtain jet Richardson number of 0.02 and 0.008 , respectively, in Earth gravity. The buoyancy is generally neglected for non-dimensional parameter $P=\left[\left(\frac{g d}{U_{j}^{2}}\right)^{0.5}\left(\frac{\rho_{j}}{\rho_{\infty}}\right)^{-0.25} \frac{z}{d}\right]<0.5$ (Ricou and Spalding, 1961;

Takahashi et al., 1982). In this study, $\mathrm{P}$ was computed using the normalized transition length measured in Earth gravity to represent $\mathrm{z} / \mathrm{d}$. For $\mathrm{Ri}=0.02, \mathrm{z} / \mathrm{d}=4.0$ and hence, $\mathrm{P}=0.37$. For $\mathrm{Ri}=0.008$, $\mathrm{z} / \mathrm{d}$ was 4.8 , resulting in $\mathrm{P}=0.28$. Thus, according to the literature, both jets of this study are considered momentum-dominated (or inertial) with negligible effects of buoyancy. Results presented next will show that this is not the case because both the flow structure and transition behavior are affected by buoyancy.

\section{Flow Visualization}

Figure 1 shows rainbow schlieren images (reproduced in black and white) for the two test cases in Earth gravity and microgravity. The injector wall and exit are visible as a black region in the upstream portion of images. For case 1, the flow transition in Earth gravity, indicated by the appearance of smaller scale structures at the jet center, is evident 
in Fig. 1.1(a) approximately at $\mathrm{z} / \mathrm{d}=3.8$. According to Fig. 1.1(b), the transition is not visible in the field-of-view in microgravity. The jet remains laminar in the absence of gravitational forcing, providing direct evidence of buoyancy effects in an inertial jet. Figures 1.2 (a) and (b) for case 2 show that transition occurs approximately at $\mathrm{z} / \mathrm{d}=4.8$ in Earth gravity and at $\mathrm{z} / \mathrm{d}=5.5$ in microgravity. Again, the lack of buoyancy has extended the laminar coherent region in microgravity. Comparing Earth gravity images in Figure 1, an increase in transition length at the lower jet Richardson number is observed. Results clearly show that buoyancy is important in low-density gas jets at $\mathrm{Ri}<0.02$.

\section{Space-Time Images}

The transient flow behavior is visualized from space-time images in Fig. 2, developed by concatenating $0.1-\mathrm{sec}$ of data at specified axial planes from a sequence of 100 schlieren images. Images are shown for case 1 at $\mathrm{z} / \mathrm{d}=4.4$ and case 2 at $\mathrm{z} / \mathrm{d}=5.4$ to represent the transitional/turbulent regions in Earth gravity. For case 1, the periodicity of the flow and breakdown of the potential core in Earth gravity is evident in Fig. 2.1(a). Note that the smaller scale structures have reached the jet center. In microgravity (Fig. $2.1 \mathrm{~b}$ ), the jet center is uncontaminated although periodic oscillations are observed for $0.4<\mathrm{r} / \mathrm{d}<0.8$. The transition is absent since the flow is laminar at this axial location in microgravity. For case 2, the Earth gravity image in Fig. 2.2(a) shows repeatable structures contaminating the jet center. The structures in microgravity are significantly different as shown by the image in Fig. 2.2(b). Overall, the images in Fig. 2 reveal differences in the temporal behavior of Earth gravity and microgravity jet flows to highlight the underlying effects of buoyancy. 


\section{Oscillating Flow Characteristics}

Fast Fourier Transform (FFT) analysis of angular deflection data obtained from an ensemble of 256 images each in Earth gravity and microgravity was performed to quantitatively assess buoyancy effects on jet oscillations. Figure 3.1(a) shows the power spectra in the transition region $(\mathrm{z} / \mathrm{d}=4.4, \mathrm{r} / \mathrm{d}=0.5)$ for case 1 in Earth gravity. The dominant frequency of $120 \mathrm{~Hz}$ was the same as that measured in the upstream laminar regime. Two smaller peaks representing sub-harmonics at $240 \mathrm{~Hz}$ and $360 \mathrm{~Hz}$ are also present, signifying the breakdown of large-scale structures in the transition region. In microgravity (Fig. 3.1b), the oscillation frequency is the same (i.e., 120Hz) although the spectral power has decreased. Matching spectral behavior in Earth and microgravity indicates that the flow may not have fully adjusted to microgravity within the 2.2-sec drop period. Longer duration microgravity (or zero gravity) experiments are desired to eliminate residual effects of Earth gravity, especially in the low-momentum regions of the jet. Figure 3.2(a)-(b) show the power spectra in the transition region $(\mathrm{z} / \mathrm{d}=5.4, \mathrm{r} / \mathrm{d}=$ 0.5 ) for case 2. A dominant frequency of $215 \mathrm{~Hz}$ is observed both in Earth gravity and microgravity. Sub-harmonics at higher frequencies were not captured because of the temporal resolution limitation. The spectral power has diminished in microgravity, signifying the importance of buoyancy on flow transition.

\section{CONCLUSIONS}

Experiments were performed in Earth gravity and microgravity to evaluate buoyancy effects on inertial helium jets at Richardson numbers of 0.02 and 0.008 and jet Reynolds number of 800 . Schlieren images show visual differences in the flow transition behavior in Earth gravity and microgravity. Lack of buoyancy delayed flow transition in 
microgravity. Space-time images and spectral plots provide evidence of significant buoyancy effects on flow oscillation and transition in inertial, low-density gas jets.

\section{ACKNOWLEDGEMENTS}

This work was supported by Physical Sciences Division of NASA's Office of Biological and Physical research under grant NAG 3-2388.

\section{REFERENCES}

Kyle DM, Sreenivasan KR (1993) The instability and breakdown of a round variabledensity Jet, J Fluid Mech. 249:619-664.

Pasumarthi KS (2004) Buoyancy effects on flow structure and instability of lowdensity gas jet, PhD Dissertation, University of Oklahoma.

Richards CD, Breuel BD, Clark RP, Troutt TR (1996) Concentration measurements in a self-excited jet, Exp Fluids 21:103-109.

Ricou FP, Spalding DB (1961) Measurement of entrainment by axisymmetrical turbulent jets, J Fluid Mech. 11:21-32.

Subbarao ER, Cantwell BJ (1992) Investigation of a co-flowing buoyant jet: experiments on the effects of Reynolds number and Richardson number, J Fluid Mech. 245:69-90.

Takahashi F, Mizomoto M, Ikai S (1982) Transition from laminar to turbulent free jet diffusion flames, Combust Flame 48:85-95.

Yildirim BS, Agrawal AK (2004) Full field measurements of self-excited oscillations in momentum-dominated helium jets, Exp Fluids, in review. 


\section{LIST OF FIGURES}

Figure 1. Rainbow schlieren images in Earth gravity (left) and microgravity (right). 1(a)-(b): Case 1 ( $\mathrm{Ri}=0.02) ; 2(\mathrm{a})-(\mathrm{b})$ : Case $2(\mathrm{Ri}=0.008)$

Figure 2. Spatio-temporal images in Earth gravity (left) and microgravity (right).

1(a)-(b): Case 1 ( $\mathrm{Ri}=0.02), \mathrm{z} / \mathrm{d}=4.4 ; 2(\mathrm{a})-(\mathrm{b})$ : Case 2, $\mathrm{z} / \mathrm{d}=5.4(\mathrm{Ri}=0.008)$

Figure 3. Power spectra of angular deflection data in Earth gravity (left) and microgravity (right) for $\mathrm{r} / \mathrm{d}=0.5$. 1: Case $1(\mathrm{Ri}=0.02) ; 2$ : Case $2(\mathrm{Ri}=0.008)$ 

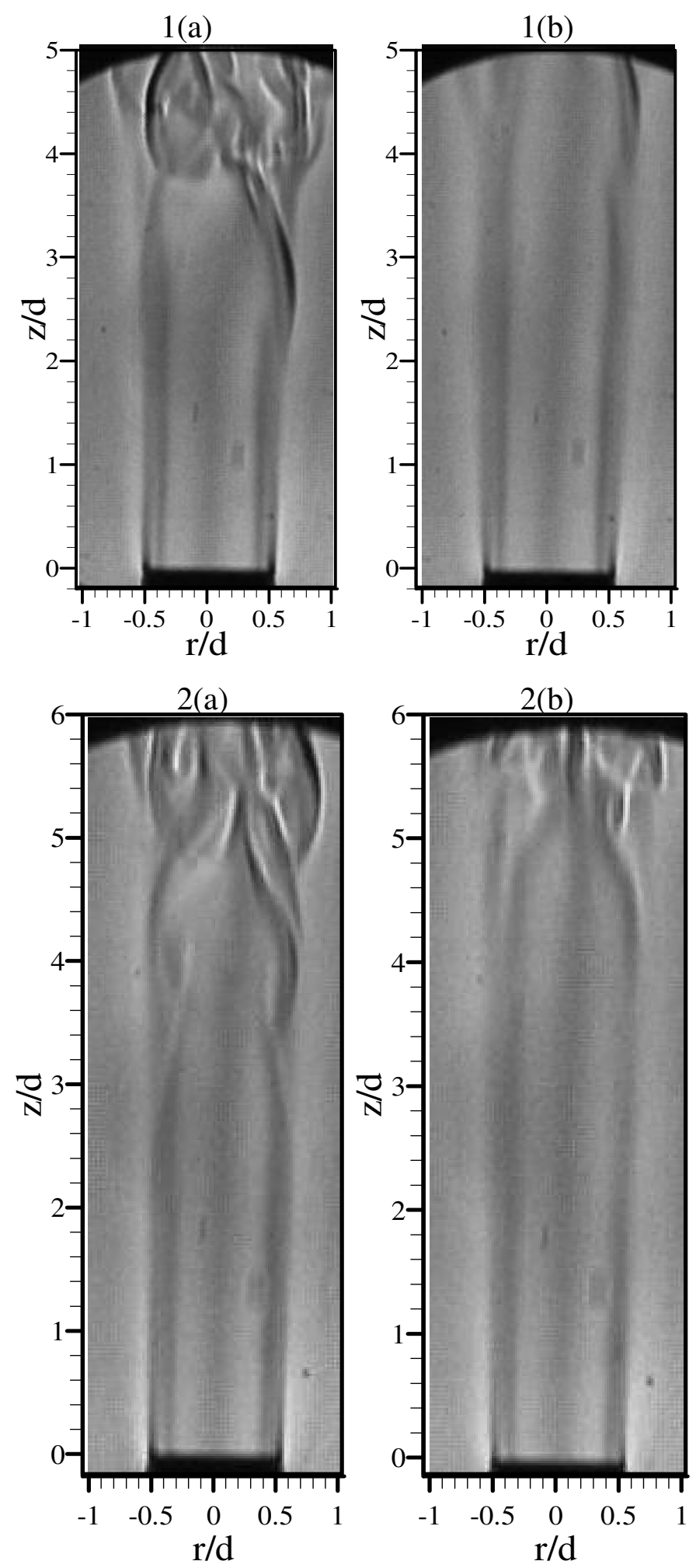

Figure 1. 

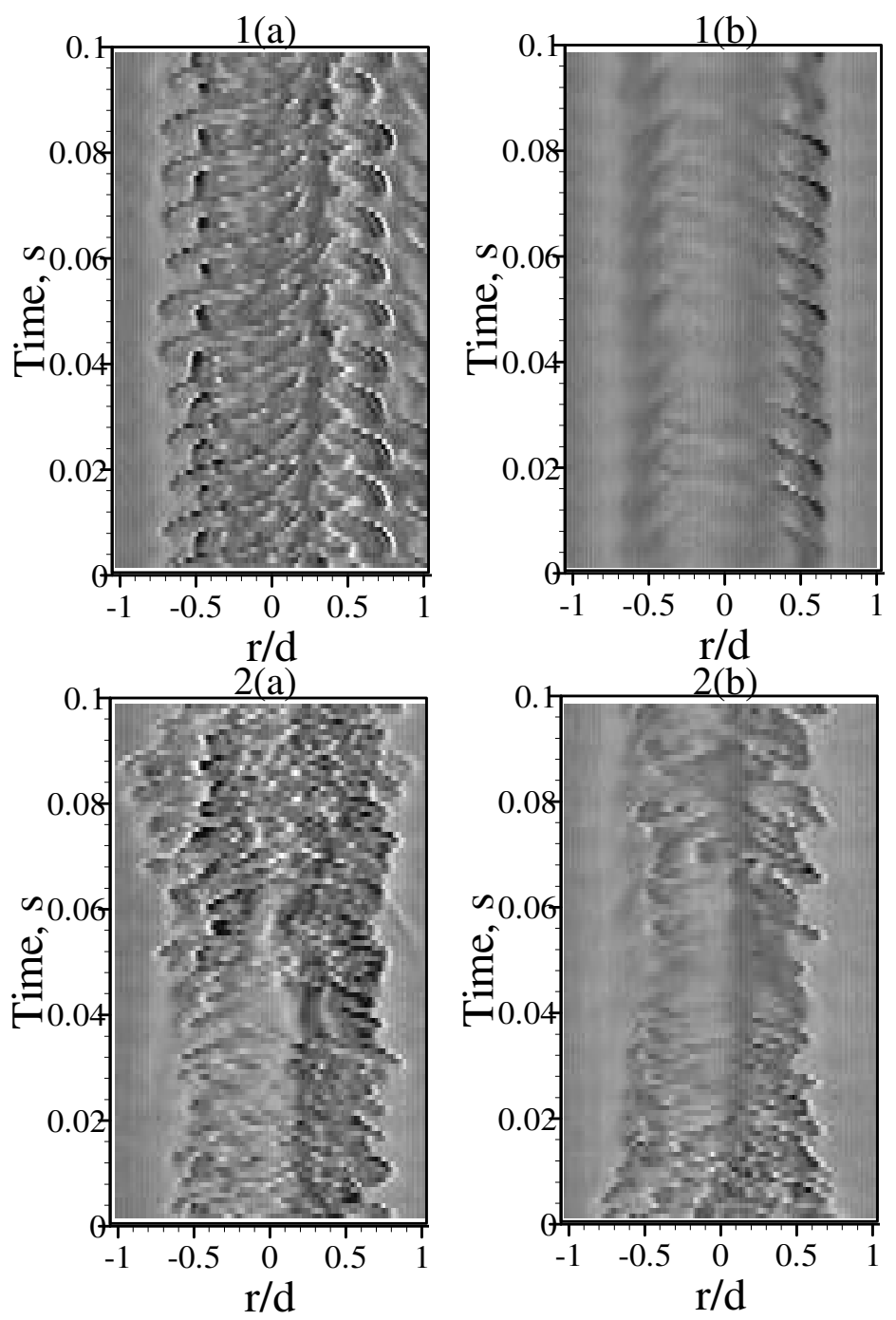

Figure 2. 


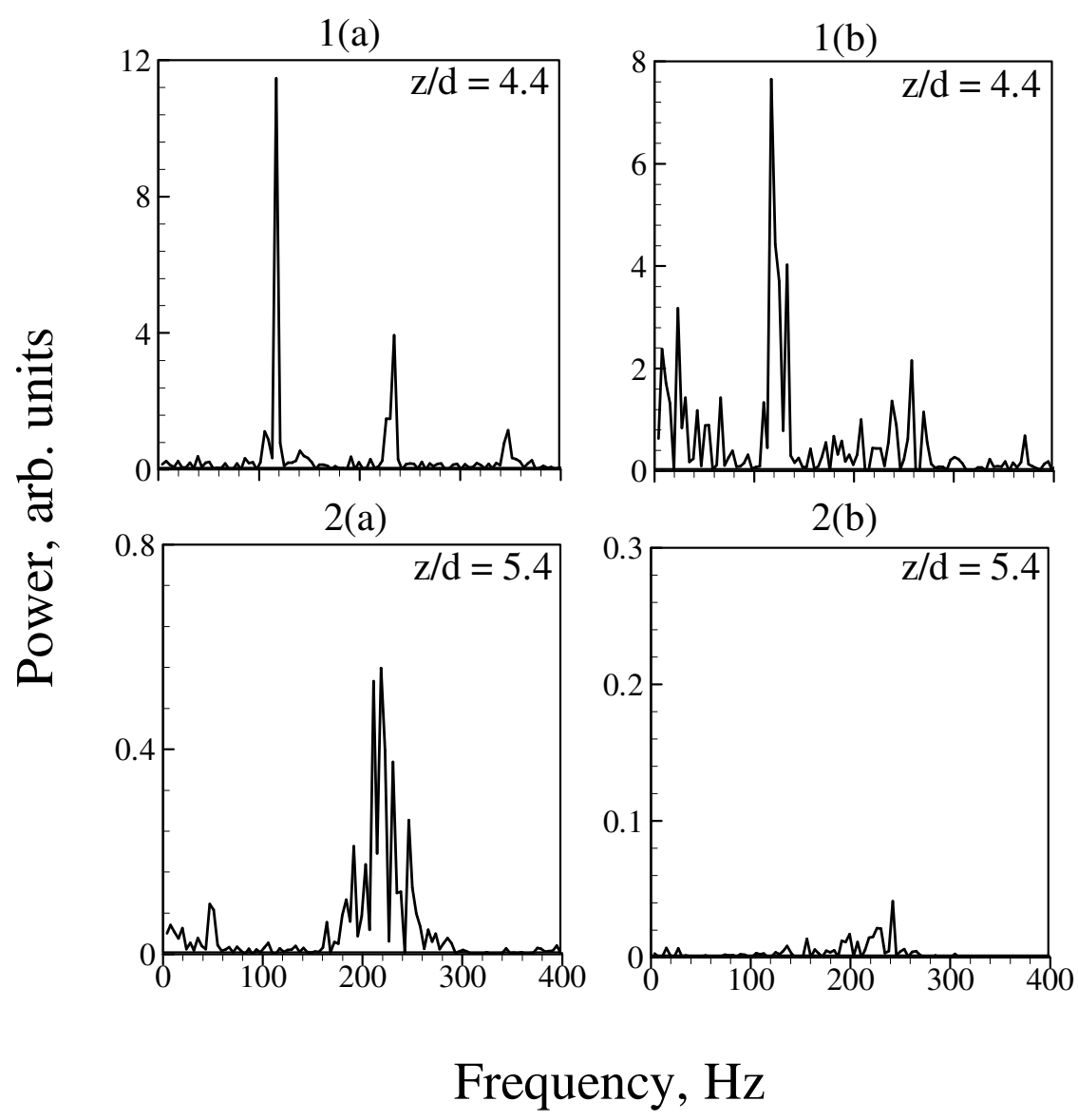

Figure 3. 